\title{
Adaptive Artificial Time Delay Control for Bipedal Walking with Robustification to State-dependent Constraint Forces
}

\author{
Amisha Bhaskar ${ }^{1}$, Swati Dantu ${ }^{2}$, Spandan Roy ${ }^{2}$, Jinoh Lee ${ }^{3}$ and Simone Baldi ${ }^{4}$
}

\begin{abstract}
Long standing challenges in adaptive bipedal walking control (i.e. control taking care of unknown robot parameters) were to unify the control design instead of designing multiple controllers for different walking phases as well as to bypass computing constraint forces, since it often leads to complex designs. A few attempts to design a single controller for all walking phases ignored or oversimplified the constraint forces. However, these forces are state-dependent and may lead to conservative performance or instability if not countered properly. This work proposes an innovative adaptive control method, based on artificial time delay control, which covers the entire bipedal walking phase and provides robustness against state-dependent unmodelled dynamics such as constraint forces and external impulsive forces arising during walking. Studies using a high fidelity simulator under various forms of disturbances show the effectiveness of the proposed design over the state of the art.
\end{abstract}

\section{INTRODUCTION}

Owing to their capability to work in diverse applications like humans, biped robots have attained a great amount of research interest in the past decades [1]-[3]. Successful bipedal walking demands accurate tracking of desired leg motion [4]. However, a biped dynamics is usually complex, involves multiple degrees-of-freedom (DoF) and highly nonlinear in nature [5]. Consequently, the research on control of bipedal walking has inevitably looked beyond the model based control strategies [4], [6]-[8] and toward adaptive control [9]-[12] to tackle limited parametric knowledge.

Artificial time delay based control is a control strategy requiring limited system knowledge: it was proposed in [13], [14], by approximating uncertainties via input and state information of immediate past instant. Owing to its simplicity in implementation and significantly low computation burden, adaptive control using artificial delay found remarkable acceptance in the robotics community in the last decade [15]-[19], including bipedal robot control [20]-[22].

This work was partly supported by the IIIT-H seed grant no. 19-20/003, by ITECH R\&D program of MOTIE/KEIT (No. 20014398 Development of a kinematically versatile, easy-to-use, safety-supported, AI-integrable, and high speed ( $\geq 5 \mathrm{kHz}$ ) robot controller.), and by Double Innovation Plan Grant 4207012004, the Special Funding for Overseas talents Grant 6207011901. The first two authors contributed equally.

${ }^{1}$ A. Bhaskar is with Netaji Subhas University of Technology, New Delhi, India (amishab.mp18@nsut.ac.in)

2 S. Dantu and S. Roy are with Robotics Research Center, International Institute of Information Technology Hyderabad (IIIT-H), Hyderabad, India (swati.dantueresearch.iiit.ac.in, spandan.royeiit.ac.in)

3 J. Lee is with Institute of Robotics and Mechatronics, German Aerospace Center (DLR), Germany (jinoh. lee@dlr.de)

${ }^{4} \mathrm{~S}$. Baldi is with School of CyberScience and Engineering, Southeast University, Nanjing, China and guest with Delft Center for Systems and Control, TU Delft, The Netherlands (s.baldi@tudelft.nl)
Depending on the model used for control design, literature of bipedal walking control can be broadly classified under two approaches: (i) one relies on unconstrained leg dynamics (cf. [4], [22]) and (ii) another relies on multi-model dynamics (cf. [6]-[10]) accounting for various stages of walking (e.g., single support stage, double support stage (cf. [5] for the definitions). Despite being easier for control design, the first approach ignores the constraint forces when both feet are on ground stemming from the dual support stage; the second approach, however, considers different dynamics for each walking stages requiring either a different individual controller [6]-[8] for each walking stage or relatively complicated control design to solve/estimate for the constraint forces [9], [10]. Considering the individual pros and cons of these two approaches, a potential solution can be to design a controller based on the first approach, but considering the constraint forces as unknown/unmodelled dynamics.

Therefore, based on the proven benefits of artificial time delay based designs over other adaptive schemes, an obvious question arises: can the state-of-the-art biped controllers [4], [22] be extended to handle the unmodelled constraint forces? Unfortunately, we do not get a positive answer for this question as constraint forces are state-dependent terms and cannot be bounded a priori [6]-[8]; whereas, [4], [22] cannot handle state-dependent unknown uncertainties (cf. [23] and discussion later in Remark 4). In fact, under such uncertainty setting, instability cannot be ruled out for adaptive controllers which are built on the assumption of a priori bounded uncertainty (cf. [24]).

In view of the above discussion, an adaptive-robust TDE (ARTDE) scheme is designed for bipedal walking with the following contributions:

- The proposed controller is simpler as it does not require different controllers for different phases of walking motion (cf. [6]-[8]) or does not require to compute constraint forces separately (cf. [9], [10]).

- Unlike [20]-[22], the proposed adaptive TDE method is also robust (hence called adaptive-robust TDE) to constraint forces, which are considered as state-dependent unmodelled dynamics.

Further, extensive study using high fidelity simulator has shown remarkable accuracy of ARTDE over the state-of-theart during bipedal walking motion.

The following notations are used in the paper: $(\bullet)_{L}$ denotes that $(\bullet)(t)$ is delayed as $(\bullet)(t-L) ;\|\bullet\|$ denotes 2 -norm and I denotes Identity matrix. 


\section{System Dynamics AND Problem Formulation}

Let us consider the following class of $n$ degrees-offreedom (DOFs) dynamics for the biped robot [4], [22]

$$
\mathbf{M}(\mathbf{q}) \ddot{\mathbf{q}}+\mathbf{H}(\mathbf{q}, \dot{\mathbf{q}})=\tau,
$$

where $\mathbf{q} \in \mathbb{R}^{n}$ is the joint position; $\tau \in \mathbb{R}^{n}$ is the control input; $\mathbf{M}(\mathbf{q}) \in \mathbb{R}^{n \times n}$ is the mass/inertia matrix and $\mathbf{H}(\mathbf{q}, \dot{\mathbf{q}}) \in$ $\mathbb{R}^{n}$ combines other system dynamics terms (e.g., Coriolis, friction, gravity) including unmodelled dynamics and disturbances. The following standard property holds

Property ([4], [20]-[22]): The matrix $\mathbf{M}(\mathbf{q})$ is uniformly positive definite for all $\mathbf{q}$, i.e., $\exists \psi_{1}, \psi_{2} \in \mathbb{R}^{+}$such that

$$
\psi_{1} \mathbf{I} \leq \mathbf{M}(\mathbf{q}) \leq \psi_{2} \mathbf{I} \Rightarrow\left(1 / \psi_{2}\right) \mathbf{I} \leq \mathbf{M}^{-1}(\mathbf{q}) \leq\left(1 / \psi_{1}\right) \mathbf{I}
$$

Remark 1 (On the system dynamics): As an alternative to the unconstrained system dynamics (1), some researchers have proposed constrained multi-modal dynamics (cf. [6][10]). However, the latter approach involves different dynamics for different walking phases (e.g., single support, double support, impulse etc.), requiring different controller for each phases, making the control design and analysis comparatively difficult [8]. Dynamics (1) can simplify the control design provided it can handle the ground reaction forces acting as impulsive unmodelled dynamics.

Assumption 1 (Uncertainty setting): Inertia matrix $\mathbf{M}$ is not precisely known, but its upper bound $\psi_{2}$ from (2) is known. At the same time, the state-dependent (via $\mathbf{q}$ and $\dot{\mathbf{q}}$ ) dynamics term $\mathbf{H}$ in (1) is unknown. Hence, $\mathbf{H}$ cannot be considered to be bounded by a constant a priori to control design [24], [25].

Remark 2 (Generality of the proposed approach):

Bipedal motion from a constrained and an unconstrained dynamics are related via state-dependent dynamics terms [8]. Therefore, Assumption 1 allows to include constraint forces in the unified dynamics (1) as unmodelled dynamics. Nevertheless, state-of-the-art adaptive designs cannot handle such a challenge for biped walking problem (cf. Remark 4).

Assumption 2: The desired trajectory $\mathbf{q}^{d}(t)$ is designed such that $\mathbf{q}^{d}, \dot{\mathbf{q}}^{d}, \ddot{\mathbf{q}}^{d} \in \mathscr{L}_{\infty}$. Further, to avoid kinematic singularity, the desired knee angle trajectory is designed such that the knee is never fully-stretched (cf. [4]).

In view of the aforementioned discussion, the control problem is defined as below:

Control Problem: Under Assumptions 1-2 and Property (2), design an adaptive control for biped walking motion while negotiating unknown state-dependent uncertainty stemming from constraint forces (in line with Remark 2).

\section{Controller Design And Analysis}

Before presenting the proposed controller, the biped dynamics (1) is re-arranged by introducing a constant and positive definite diagonal matrix $\overline{\mathbf{M}}$ as

$$
\begin{array}{ll} 
& \overline{\mathbf{M}} \ddot{\mathbf{q}}+\mathbf{N}(\mathbf{q}, \dot{\mathbf{q}}, \ddot{\mathbf{q}})=\tau, \\
\text { with } & \mathbf{N}(\mathbf{q}, \dot{\mathbf{q}}, \ddot{\mathbf{q}})=[\mathbf{M}(\mathbf{q})-\overline{\mathbf{M}}] \ddot{\mathbf{q}}+\mathbf{H}(\mathbf{q}, \dot{\mathbf{q}})
\end{array}
$$

and the choice of $\overline{\mathbf{M}}$ is discussed later (cf. discussion after (18)). Note that owing to Assumption 1, the unknown dynamics is now subsumed under $\mathbf{N}$.

Let us define the tracking error as $\mathbf{e}(t)=\mathbf{q}^{d}(t)-\mathbf{q}(t)$. Subsequently, variable dependency will be omitted whenever obvious for brevity. The control input $\tau$ is designed as

$$
\begin{aligned}
\tau & =\overline{\mathbf{M}} \mathbf{u}+\hat{\mathbf{N}}(\mathbf{q}, \dot{\mathbf{q}}, \ddot{\mathbf{q}}), \\
\mathbf{u} & =\mathbf{u}_{0}+\Delta \mathbf{u}, \\
\mathbf{u}_{0} & =\ddot{\mathbf{q}}^{d}+\mathbf{K}_{D} \dot{\mathbf{e}}+\mathbf{K}_{P} \mathbf{e},
\end{aligned}
$$

where $\mathbf{K}_{P}, \mathbf{K}_{D} \in \mathbb{R}^{n \times n}$ are two positive definite matrices; $\Delta \mathbf{u}$ is the adaptive control term to be designed later and $\hat{\mathbf{N}}$ is the estimated value of $\mathbf{N}$ computed via the past input and state data as [13], [14]

$$
\hat{\mathbf{N}}(\mathbf{q}, \dot{\mathbf{q}}, \ddot{\mathbf{q}}) \cong \mathbf{N}\left(\mathbf{q}_{L}, \dot{\mathbf{q}}_{L}, \ddot{\mathbf{q}}_{L}\right)=\tau_{L}-\overline{\mathbf{M}} \ddot{\mathbf{q}}_{L},
$$

where $L>0$ is a small time delay.

Remark 3 (Artificial time delay): The uncertainty estimating process via past data (i.e., time delayed data) as in (6) is typically called in literature as time-delay estimation (TDE) or artificial delay based estimation method, since time delay is invoked into the system artificially/intentionally via past data, while the original system was free of any time delay. Since the TDE process (6) relies on immediate past data, $L$ is typically selected in practice as the sampling interval of available hardware [13]-[19].

Substituting (5a) in (3), one obtains

$$
\ddot{\mathbf{e}}=-\mathbf{K}_{D} \dot{\mathbf{e}}_{L}-\mathbf{K}_{P} \mathbf{e}_{L}+\sigma,
$$

where $\sigma=\overline{\mathbf{M}}^{-1}(\mathbf{N}-\hat{\mathbf{N}})$ represents the estimation error stemming from (6), also termed as TDE error.

Design of the adaptive control term $\Delta \mathbf{u}$ relies on the upper bound structure of $\sigma$ as derived subsequently, followed by the proposed adaptive law.

\section{A. Upper bound structure of $\sigma$}

From (4) and (7), the following relations can be achieved:

$$
\begin{aligned}
\hat{\mathbf{N}} & =\mathbf{N}_{L}=\left[\mathbf{M}\left(\mathbf{q}_{L}\right)-\overline{\mathbf{M}}\right] \ddot{\mathbf{q}}_{L}+\mathbf{H}_{L}, \\
\sigma & =\ddot{\mathbf{q}}-\mathbf{u} .
\end{aligned}
$$

Using (8), the control input $\tau$ in (5a) can be rewritten as

$$
\tau=\overline{\mathbf{M}} \mathbf{u}+\left[\mathbf{M}\left(\mathbf{q}_{L}\right)-\overline{\mathbf{M}}\right] \ddot{\mathbf{q}}_{L}+\mathbf{H}_{L} .
$$

Multiplying both sides of (9) with $\mathbf{M}$ and using (1) and (10) we have

$$
\begin{aligned}
\mathbf{M} \sigma & =\tau-\mathbf{H}-\mathbf{M u}, \\
& =\overline{\mathbf{M}} \mathbf{u}+\left[\mathbf{M}\left(\mathbf{q}_{L}\right)-\overline{\mathbf{M}}\right] \ddot{\mathbf{q}}_{L}+\mathbf{H}_{L}-\mathbf{H}-\mathbf{M u} .
\end{aligned}
$$

Defining $\mathbf{K} \triangleq\left[\mathbf{K}_{P} \mathbf{K}_{D}\right]$ and using (7) we have

$$
\ddot{\mathbf{q}}_{L}=\ddot{\mathbf{q}}_{L}^{d}-\ddot{\mathbf{e}}_{L}=\ddot{\mathbf{q}}_{L}^{d}+\mathbf{K} \xi_{L}-\sigma_{L}+\Delta \mathbf{u}_{L} .
$$

Substituting (12) into (11), and after re-arrangement yields

$$
\sigma=\underbrace{\mathbf{M}^{-1} \overline{\mathbf{M}}\left(\Delta \mathbf{u}-\Delta \mathbf{u}_{L}\right)}_{\chi_{1}}+\underbrace{\mathbf{M}^{-1}\left(\mathbf{M}_{L} \Delta \mathbf{u}_{L}-\mathbf{M} \Delta \mathbf{u}\right)}_{\chi_{2}}
$$




$$
\begin{aligned}
& +\underbrace{\mathbf{M}^{-1}\left\{\overline{\mathbf{M}} \ddot{\mathbf{q}}^{d}-\left(\mathbf{M}-\mathbf{M}_{L}+\overline{\mathbf{M}}\right) \ddot{\mathbf{q}}_{L}^{d}+\mathbf{H}_{L}-\mathbf{H}\right\}}_{\chi_{3}} \\
& +\underbrace{\mathbf{M}^{-1}\left(\mathbf{M}_{L}-\overline{\mathbf{M}}\right) \mathbf{K} \xi_{L}}_{\chi_{4}}-\underbrace{\mathbf{M}^{-1}\left(\mathbf{M}_{L}-\overline{\mathbf{M}}\right) \sigma_{L}}_{\chi_{5}} \\
& +\underbrace{\left(\mathbf{M}^{-1} \overline{\mathbf{M}}-\mathbf{I}\right) \mathbf{K} \xi}_{\chi_{6}} .
\end{aligned}
$$

The following Lemma provides the upper bound of $\|\sigma\|$ :

Lemma 1 ([23], [26]): Under the condition

$$
\|\mathbf{E}\|=\| \mathbf{I}-\mathbf{M}^{-1}(\mathbf{q}) \overline{\mathbf{M}}||<1,
$$

and the property (2), there exist (unknown) scalars $\delta_{1,2, \cdots, 5}$, such that

$$
\begin{aligned}
\left\|\chi_{1,2,3}\right\| & \leq \delta_{1,2,3},\left\|\chi_{4}\right\| \leq\left\|\mathbf { E K } \left|\||| \xi\|+\delta_{4}\right.\right. \\
\left\|\chi_{5}\right\| & \leq\left\|\mathbf { E } \left|\||| \sigma\|+\delta_{5},\left\|\chi_{6}\right\| \leq\|\mathbf{E K} \mid\| \xi \|\right.\right.
\end{aligned}
$$

yielding

$$
\begin{aligned}
& \qquad \sigma\left\|\leq \beta_{0}+\beta_{1}\right\| \xi \|, \\
& \text { where } \beta_{0}=\frac{\sum_{i=1}^{5} \delta_{i}}{1-\|\mathbf{E}\|}, \beta_{1}=\frac{2\|\mathbf{E K}\|}{1-\|\mathbf{E}\|}
\end{aligned}
$$

The condition (14), which is standard in the literature of TDE based controllers [13]-[22], gives the criterion to select $\overline{\mathbf{M}}$, which is feasible since upper bound knowledge of $\mathbf{M}$ is available from Assumption 1.

Remark 4 (State-dependent TDE error bound): The upper bound structure of TDE error $\sigma$ in (17) has statedependency via $\beta_{1}\|\xi\|$, implying $\sigma$ cannot be considered bounded a priori. We will show later that assuming a priori boundedness not only sacrifices tracking accuracy, but may cause instability during bipedal walking (cf. Sect. IV.B).

\section{B. Design of the Adaptive Control Law $\Delta \mathbf{u}$}

The term $\Delta \mathbf{u}$ is designed as

$$
\Delta \mathbf{u}=\alpha c \operatorname{sig}(\mathbf{s}, \varepsilon),
$$

where $\mathbf{s}=\mathbf{B}^{T} \mathbf{P} \xi, \xi=\left[\begin{array}{ll}\mathbf{e}^{T} & \dot{\mathbf{e}}^{T}\end{array}\right]^{T}$ and $\mathbf{P}>\mathbf{0}$ is the solution of the Lyapunov equation $\mathbf{A}^{T} \mathbf{P}+\mathbf{P A}=-\mathbf{Q}$ for some $\mathbf{Q}>\mathbf{0}$, where $\mathbf{A}=\left[\begin{array}{cc}\mathbf{0} & \mathbf{I} \\ -\mathbf{K}_{P} & -\mathbf{K}_{D}\end{array}\right], \mathbf{B}=\left[\begin{array}{l}\mathbf{0} \\ \mathbf{I}\end{array}\right] ; \alpha \in \mathbb{R}^{+}$is a userdefined scalar; $c \in \mathbb{R}^{+}$is the overall switching gain tackling $\sigma$ and $\operatorname{sig}(\mathbf{s}, \varepsilon) \triangleq \mathbf{s} / \sqrt{\|\mathbf{s}\|^{2}+\varepsilon}$. Here, $\varepsilon$ is a small positive scalar used to avoid chattering.

The switching gain $c$ in (19) is formulated based on the structure of $\|\sigma\|$ as

$$
c=\hat{\beta}_{0}+\hat{\beta}_{1}\|\xi\|,
$$

where $\hat{\beta}_{0}, \hat{\beta}_{1}$ are the estimates of $\beta_{0}, \beta_{1} \in \mathbb{R}^{+}$, respectively. The gains are evaluated as follows:

$$
\dot{\hat{\beta}}_{j}=\left\{\begin{array}{ll}
\gamma_{j}\|\xi\|^{j}\|\mathbf{s}\|, & \text { if any } \hat{\beta}_{j} \leq \underline{\beta}_{j} \text { or } \mathbf{s}^{T} \dot{\mathbf{s}}>0 \\
-\gamma_{j}\|\xi\|^{j}\|\mathbf{s}\|, & \text { if } \mathbf{s}^{T} \dot{\mathbf{s}} \leq 0 \text { and all } \hat{\beta}_{j}>\underline{\beta}_{j}
\end{array},\right.
$$

with $\hat{\beta}_{j}(0) \geq \underline{\beta}_{j}>0, j=0,1$ are user-defined scalars.

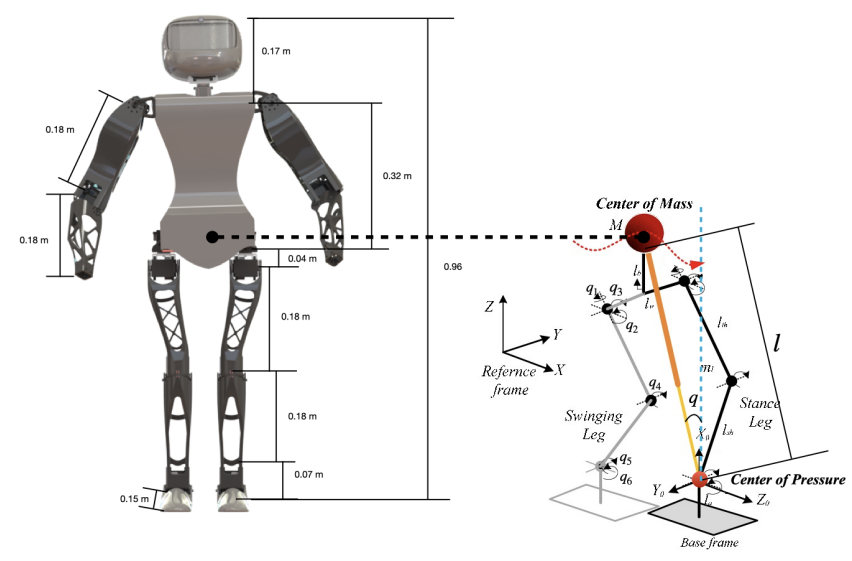

Fig. 1. Schematic of Ojas (proposed humanoid).

Combining (5a), (5b), (6) and (19), ARTDE becomes

$$
\begin{aligned}
\tau & =\underbrace{\tau_{L}-\overline{\mathbf{M}} \ddot{\mathbf{q}}_{L}}_{\text {TDE part }}+\underbrace{\overline{\mathbf{M}}\left(\ddot{\mathbf{q}}^{d}+\mathbf{K}_{D} \dot{\mathbf{e}}+\mathbf{K}_{P} \mathbf{e}\right)}_{\text {Desired dynamics injection part }} \\
& +\underbrace{\alpha \overline{\mathbf{M}} c \operatorname{sig}(\mathbf{s}, \boldsymbol{\varepsilon})}_{\text {Adaptive-robust control part }}
\end{aligned}
$$

Remark 5: In (17), the term $\beta_{0}$ can capture the effects of bounded external impulsive forces, while $\beta_{1}\|\xi\|$ can capture state-dependent unmodelled dynamics, particularly, constraint forces (along with uncertain system parameters). Therefore, compared to [22], estimating these parameters via the adaptive laws (21) helps the proposed design avoid design complexities computing constraint forces separately.

The closed-loop stability is stated via the following Theorem:

Theorem 1: The system (1) employing ARTDE with the controller (22) and adaptive law (20)-(21) is Uniformly Ultimately Bounded (UUB).

Proof: The analysis is analogous to [26] and is not reported for lack of space.

\section{Verification of the Proposed ARTDE}

\section{A. Simulation Setup}

To verify the performance of the proposed controller, a 20 DoFs humanoid named Ojas (cf. Fig. 1 for detailed mechanical structure), weighing $8.986 \mathrm{~kg}$ and $0.94 \mathrm{~m}$ tall from feet to head, is designed: each leg and arm of the robot has 6 and 4 DoFs, respectively. We relied on the high fidelity simulator Pybullet for verification of the proposed controller.

The objective is Ojas should maintain a desired walking motion in the face of various uncertainties. For this purpose, only the 6 joints of each leg, 3 hip joints, 1 knee joint and 2 angle joints, while other joints of the robot are kept fixed (i.e., fixed upper-body). Thus, twelve joints are operated simultaneously for both legs. Subsequently we denote $q_{1}=$ yaw hip joint, $q_{2}=$ roll hip joint, $q_{3}=$ pitch hip joint, $q_{4}=$ knee joint, $q_{5}=$ pitch ankle joint and $q_{6}=$ roll ankle joint. Following [4], the desired walking motion is generated (cf. Fig. 2) via the desired trajectories for the six joints for each leg as in Fig. 3, leading to a walking speed $0.2 \mathrm{~m} / \mathrm{s}$ with 1 s step period and stride of $0.1 \mathrm{~m}$. 


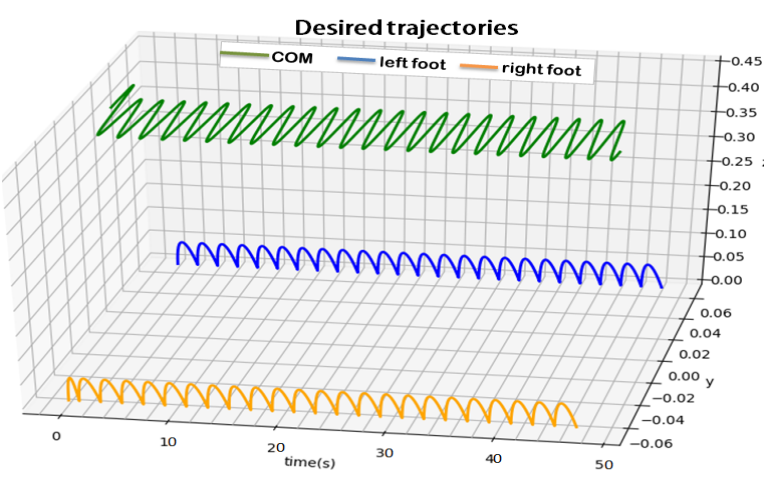

Fig. 2. Desired trajectories of Center of mass (COM) and feet.

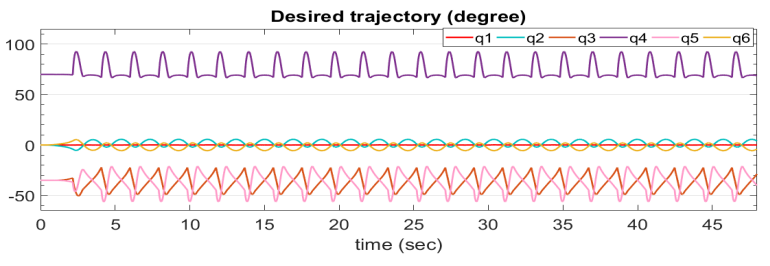

Fig. 3. Desired leg joint trajectories (same for both the legs).

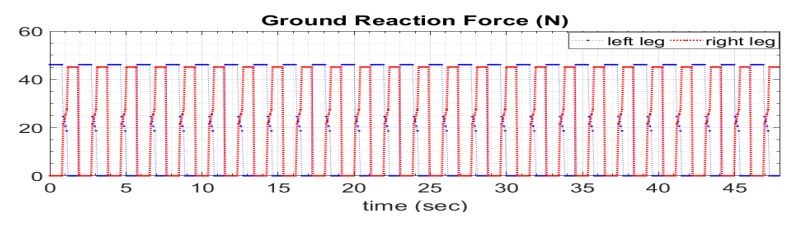

Fig. 4. Ground reaction force on the legs.

\section{B. Simulation Scenario, Results and Analysis}

To properly judge the effectiveness of the proposed ARTDE scheme against state-of-the-art, we consider the classical TDE [13] (control law (5) with $\Delta \mathbf{u}=\mathbf{0}$ ) and the adaptive TDE biped controller [22] (called ATDE henceforth), with $\Delta \mathbf{u}$ as (19) and the following adaptive law for $c$

$$
\dot{c}=\left\{\begin{array}{ll}
\gamma_{0}\|\mathbf{s}\|, & \text { if } c \leq \underline{\beta}_{0} \text { or }\left(\|\mathbf{s}\|-\left\|\mathbf{s}_{L}\right\|\right)>0 \\
-\gamma_{0}\|\mathbf{s}\|, & \text { if }\left(\|\mathbf{s}\|-\left\|\mathbf{s}_{L}\right\|\right) \leq 0
\end{array} .\right.
$$

Three simulation scenarios, S1, S2 and S3, are considered in following subsections with same control design parameters as: $\overline{\mathbf{M}}=0.042 \mathbf{I}\left(\mathrm{kgm}^{2}\right), \mathbf{K}_{P}=25 \mathbf{I}, \mathbf{K}_{D}=10 \mathbf{I}, L=0.001 \mathrm{sec}$, $\mathbf{Q}=\mathbf{I}, \boldsymbol{\varepsilon}=5 \times 10^{-5}, \alpha=4, \underline{\beta}_{j}=0.01, \hat{\beta}_{j}(0)=0.01, j=0,1$. For parity in the comparison, same values of $\overline{\mathbf{M}}, \mathbf{K}_{P}, \mathbf{K}_{D}, L$ and $\alpha, \gamma_{0}, \beta_{0}$ are selected for the TDE and ATDE (23).

For all scenarios, a ground reaction force (GRF) is created following the model [27] which acts as impulsive external disturbance whenever the foot lands on the ground while walking (cf. Fig. 4) and it is considered to be unknown for all the controllers. Due to structural symmetry in Ojas, only the results for the right leg are presented to avoid repetition.

1) Description of Scenario S1: This scenario tests the capability of various controllers to adapt to the dynamic changes in the desired walking trajectory under the GRF (cf. Fig. 4), which creates a significant nonlinearity while propagating throughout the body.


Fig. 5. Tracking error for scenario S1.

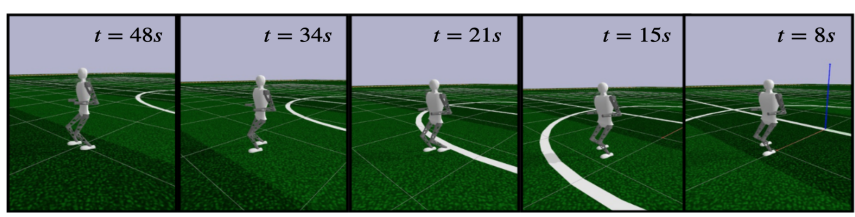

Fig. 6. The snapshots from scenario S1 with proposed controller.

Results and Discussion for S1: The tracking performance of TDE, ATDE and the proposed ARTDE are demonstrated via Fig. 5 and further collected in Table $I$ in terms of root mean squared (RMS) error, maximum absolute error (MAE) and RMS $\tau$. Spikes can be noted in every error plots whenever the GRF of the two legs overlap around their peaks (cf. Figs. 4 and 5): this indicates both the feet are on ground (double support phase) and the state-dependent constraint forces are in effect. The significantly lower peaks for ARTDE (cf. the MAE data in Table I) and minimum performance improvements of $29.1 \%$ and $20.6 \%$ in terms RMSE as compared to TDE and ATDE respectively across all the joints, clearly demonstrate its capability to handle state-dependent forces compared to others. A few snapshots of the walking motion using ARTDE is shown in Fig. 6.

2) Description of Scenario S2: In this second scenario, Ojas is required to follow the same walking motion via Fig. 3 , but now, while carrying a payload of $1.3 \mathrm{~kg}$ mass (approx. $15 \%$ of robot's mass) and using various controllers.

Results and Discussion for S2: Unlike scenario S1, Figs. 7-10 reveal that, while carrying the payload, the robot falls with TDE after 26 steps (at $t=26 \mathrm{sec}$ ) and with ATDE after 31 steps (at $t=31 \mathrm{sec}$ ) (cf. the sudden spikes in Fig. 10). Whereas, the proposed ARTDE could perform the task successfully (cf. Fig. 9). This shows that TDE and ATDE, built on the assumption of a priori bounded uncertainty, may fail in presence of state-dependent uncertainty. Since TDE and ATDE have shown destabilizing behaviour, only the performance of ARTDE is given in Table II. 




Fig. 7. The snapshots from scenario S2 of walking with payload using TDE.

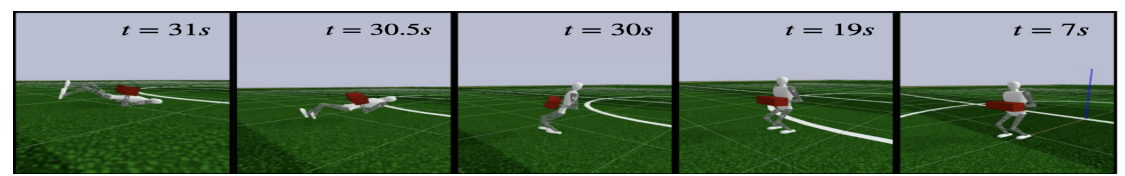

Fig. 8. The snapshots from scenario S2 of walking with payload using ATDE.

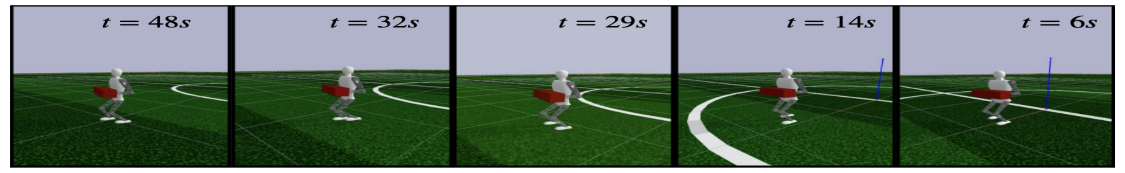

Fig. 9. The snapshots from scenario S2 of walking with payload using proposed ARTDE.

TABLE I

PERFORMANCE COMPARISON FOR SCENARIO S1

\begin{tabular}{|c|c|c|c|c|c|}
\hline \multirow{2}{*}{ Joints } & \multicolumn{3}{|c|}{ Controller } & \multicolumn{2}{|c|}{ Performance Improvement } \\
\hline & TDE & ATDE & Proposed & over TDE & over ATDE \\
\hline & \multicolumn{5}{|c|}{ RMS error (degree) } \\
\hline$q_{1}$ & 0.108 & 0.035 & 0.024 & $77.7 \%$ & $31.4 \%$ \\
\hline$q_{2}$ & 0.653 & 0.551 & 0.436 & $29.1 \%$ & $20.8 \%$ \\
\hline$q_{3}$ & 2.231 & 1.847 & 1.455 & $34.7 \%$ & $21.2 \%$ \\
\hline$q_{4}$ & 3.264 & 2.671 & 2.072 & $36.5 \%$ & $22.4 \%$ \\
\hline$q_{5}$ & 3.254 & 2.741 & 2.190 & $32.6 \%$ & $20.6 \%$ \\
\hline \multirow[t]{2}{*}{$q_{6}$} & 1.147 & 1.023 & 0.741 & $35.3 \%$ & $27.5 \%$ \\
\hline & \multicolumn{5}{|c|}{ MAE (degree) } \\
\hline$q_{1}$ & 0.173 & 0.225 & 0.075 & $56.65 \%$ & $66.67 \%$ \\
\hline$q_{2}$ & 1.841 & 0.945 & 0.746 & $59.48 \%$ & $21.06 \%$ \\
\hline$q_{3}$ & 2.456 & 1.889 & 1.447 & $41.08 \%$ & $23.40 \%$ \\
\hline$q_{4}$ & 8.7415 & 7.483 & 5.407 & $38.15 \%$ & $27.74 \%$ \\
\hline$q_{5}$ & 8.660 & 6.601 & 4.656 & $46.24 \%$ & $29.47 \%$ \\
\hline \multirow[t]{2}{*}{$q_{6}$} & 5.976 & 6.190 & 2.415 & $59.59 \%$ & $60.99 \%$ \\
\hline & \multicolumn{5}{|c|}{ RMS $\tau(\mathrm{Nm})$} \\
\hline$q_{1}$ & 2.864 & 3.076 & 2.826 & $1.33 \%$ & $8.13 \%$ \\
\hline$q_{2}$ & 3.964 & 4.176 & 4.022 & $-1.46 \%$ & $3.69 \%$ \\
\hline$q_{3}$ & 5.965 & 6.003 & 5.883 & $1.37 \%$ & $2.00 \%$ \\
\hline$q_{4}$ & 5.901 & 5.783 & 5.532 & $6.25 \%$ & $4.34 \%$ \\
\hline$q_{5}$ & 7.764 & 7.861 & 7.832 & $-2.16 \%$ & $-0.90 \%$ \\
\hline$q_{6}$ & 3.133 & 3.479 & 3.148 & $-0.48 \%$ & $9.51 \%$ \\
\hline
\end{tabular}

TABLE II

PERFORMANCE OF THE PROPOSED CONTROLLER UNDER SCENARIO S2

\begin{tabular}{cccc}
\hline \hline Joints & RMS error (degree) & MAE (degree) & RMS $\tau(\mathrm{Nm})$ \\
\hline \hline$q_{1}$ & 0.026 & 0.063 & 2.826 \\
$q_{2}$ & 0.436 & 0.747 & 4.006 \\
$q_{3}$ & 1.466 & 3.140 & 5.882 \\
$q_{4}$ & 2.120 & 5.407 & 5.657 \\
$q_{5}$ & 2.205 & 5.944 & 7.974 \\
$q_{6}$ & 0.777 & 2.795 & 3.253 \\
\hline \hline
\end{tabular}

3) Description of Scenario S3: To further verify the robustness property of the proposed ARTDE, this scenario is constructed with the following phases:

(i) In phase $1(0 \leq t<28)$, Ojas walks under similar condition of scenario $\mathrm{S} 2$.
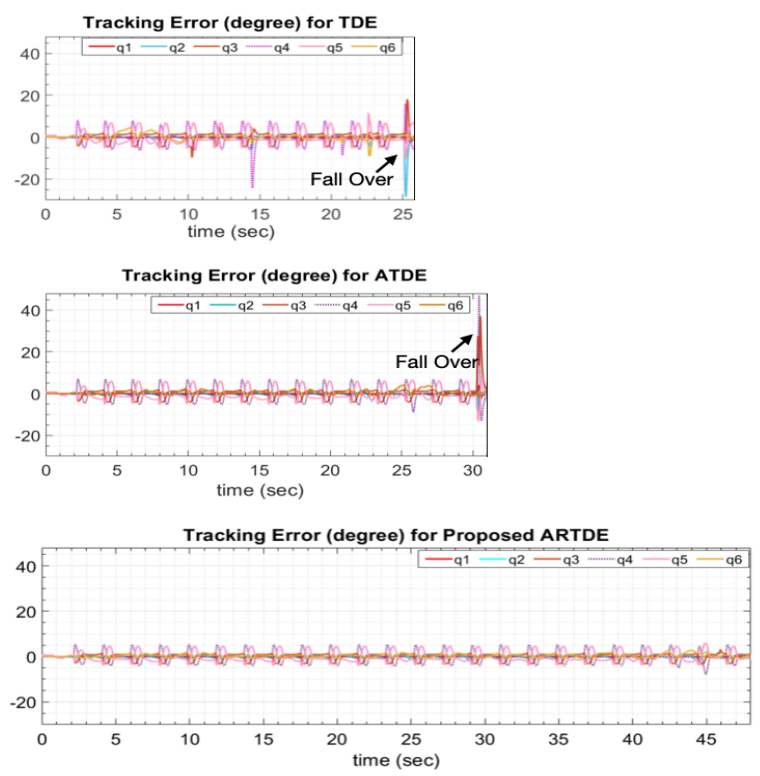

Fig. 10. TDE and ATDE fails to stabilize the robot, which falls over at $26 \mathrm{~s}$ and $31 \mathrm{~s}$ respectively.



Fig. 11. Snapshots from scenario S3.

(ii) In phase $2(28 \leq t<37)$, an external impulsive push of $10 \mathrm{~N}$ is applied on the chest (cf. second snapshot in Fig. 11) at $t=28 \mathrm{sec}$ while it was walking.

(iii) In phase $3(t \geq 37)$, another impulsive push of $10 \mathrm{~N}$ is applied at $t=37 \mathrm{sec}$ (cf. fourth snapshot in Fig. 11), 


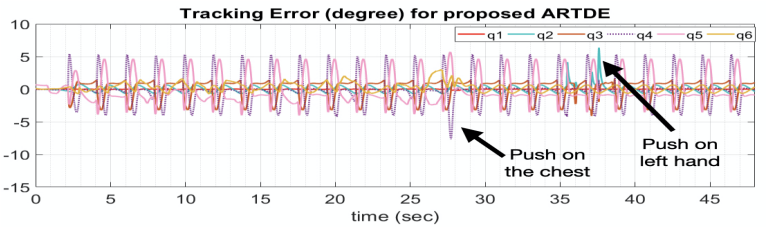

Fig. 12. Tracking error of scenario S3.

TABLE III

PERFORMANCE OF THE PROPOSED CONTROLLER UNDER SCENARIO S3

\begin{tabular}{ccccccc}
\hline \hline & \multicolumn{3}{c}{ RMS error (degree) } & \multicolumn{3}{c}{ MAE (degree) } \\
\hline Joints & Phase1 & Phase2 & Phase3 & Phase1 & Phase2 & Phase3 \\
\hline$q_{1}$ & 0.025 & 0.026 & 0.025 & 0.065 & 0.062 & 0.063 \\
$q_{2}$ & 0.436 & 0.441 & 0.497 & 0.745 & 4.118 & 6.723 \\
$q_{3}$ & 1.460 & 1.456 & 1.462 & 3.142 & 4.008 & 3.802 \\
$q_{4}$ & 2.118 & 2.320 & 2.108 & 5.361 & 7.864 & 5.853 \\
$q_{5}$ & 2.184 & 2.243 & 2.182 & 5.980 & 5.801 & 5.701 \\
$q_{6}$ & 0.763 & 0.862 & 0.770 & 2.008 & 3.061 & 2.893 \\
\hline \multicolumn{7}{c}{ RMS $\tau(\mathrm{Nm})$} \\
\hline$q_{1}$ & 2.824 & 2.912 & 2.802 & & & \\
$q_{2}$ & 3.924 & 4.004 & 4.964 & & & \\
$q_{3}$ & 5.863 & 5.912 & 5.904 & & & \\
$q_{4}$ & 5.351 & 5.801 & 5.368 & & & \\
$q_{5}$ & 7.78 & 7.981 & 7.763 & & \\
$q_{6}$ & 4.253 & 3.837 & 3.568 & & \\
\hline \hline
\end{tabular}

but now to the left arm $\left(45^{0}\right.$ to the $z$ axis).

Results and Discussion for S3: The tracking performance of ARTDE as in Fig. 12 clearly highlights the robustness of the proposed design against external disturbances. Further, comparison of RMS error in Tables II and III highlights ARTDE provides good repeatability, while higher MAE in $q_{2}$ and $q_{4}$ joints stem from the impulsive pushes.

\section{CONCLUSION}

A new artificial time delay based adaptive controller for bipedal walking was designed to effectively provide robustness against unmodelled state-dependent constraint forces and impulse forces for all bipedal walking phases. Thus, the control design and implementation became simpler compared to a multi-modal dynamics based multiple controller paradigm. Via extensive simulations under various forms of disturbances, it was shown that the state-of-art might lead to falling while walking, while the proposed controller could execute the desired walking motion with notable accuracy.

\section{REFERENCES}

[1] Q. Huang, K. Yokoi, S. Kajita, K. Kaneko, H. Arai, N. Koyachi, and K. Tanie, "Planning walking patterns for a biped robot," IEEE Trans. Rob. Autom., vol. 17, no. 3, pp. 280-289, 2001.

[2] Q. Li, Z. Yu, X. Chen, Q. Zhou, W. Zhang, L. Meng, and Q. Huang, "Contact force/torque control based on viscoelastic model for stable bipedal walking on indefinite uneven terrain," IEEE Trans. Autom. Sci. Eng., vol. 16, no. 4, pp. 1627-1639, 2019.

[3] A. Herzog, L. Righetti, F. Grimminger, P. Pastor, and S. Schaal, "Balancing experiments on a torque-controlled humanoid with hierarchical inverse dynamics," in IEEE/RSJ International Conference on Intelligent Robots and Systems, 2014, pp. 981-988.

[4] J. H. Park, "Impedance control for biped robot locomotion," IEEE Trans. Rob. Autom., vol. 17, no. 6, pp. 870-882, 2001.

[5] Y. Hurmuzlu, F. Génot, and B. Brogliato, "Modeling, stability and control of biped robots-a general framework," Automatica, vol. 40, no. 10, pp. 1647-1664, 2004.
[6] X. Mu and Q. Wu, "Development of a complete dynamic model of a planar five-link biped and sliding mode control of its locomotion during the double support phase," Int. J. Control, vol. 77, no. 8, pp. 789-799, 2004.

[7] K. Mitobe, N. Mori, Y. Nasu, and N. Adachi, "Control of a biped walking robot during the double support phase," Autonomous Robots, vol. 4, no. 3, pp. 287-296, 1997.

[8] D. J. Braun and M. Goldfarb, "A control approach for actuated dynamic walking in biped robots," IEEE Trans. Rob., vol. 25, no. 6 , pp. 1292-1303, 2009.

[9] Z. Li and S. S. Ge, "Adaptive robust controls of biped robots," IET Control Theory Appl., vol. 7, no. 2, pp. 161-175, 2013.

[10] C. Sun, W. He, W. Ge, and C. Chang, "Adaptive neural network control of biped robots," IEEE Trans. Syst. Man Cybern.: Syst., vol. 47, no. 2, pp. 315-326, 2016.

[11] L. Wang, Z. Liu, C. L. P. Chen, Y. Zhang, S. Lee, and X. Chen, "Energy-efficient svm learning control system for biped walking robots," IEEE Trans. Neural Networks Learn. Syst., vol. 24, no. 5, pp. 831-837, 2013.

[12] Z. Liu, C. Chen, Y. Zhang, and C. P. Chen, "Adaptive neural control for dual-arm coordination of humanoid robot with unknown nonlinearities in output mechanism," IEEE Trans. Cybern., vol. 45, no. 3, pp. 507$518,2014$.

[13] T. S. Hsia, T. Lasky, and Z. Guo, "Robust independent joint controller design for industrial robot manipulators," IEEE Trans. Indus. Electron. vol. 38 , no. 1, pp. 21-25, 1991.

[14] K. Youcef-Toumi and O. Ito, "A time delay controller for systems with unknown dynamics," ASME J. Dyn. Syst. Meas. Control, vol. 112, no. 1, pp. 133-142, 1990

[15] J. Lee, P. H. Chang, and R. S. Jamisola, "Relative impedance control for dual-arm robots performing asymmetric bimanual tasks," IEEE Trans. Indus. Electron., vol. 61, no. 7, pp. 3786-3796, 2014.

[16] S. Roy, S. Nandy, R. Ray, and S. N. Shome, "Time delay sliding mode control of nonholonomic wheeled mobile robot: experimental validation," in IEEE International Conference on Robotics Automation. IEEE, 2014, pp. 2886-2892.

[17] J. Baek, M. Jin, and S. Han, "A new adaptive sliding-mode control scheme for application to robot manipulators," IEEE Trans. Indus. Electron., vol. 63, no. 6, pp. 3628-3637, 2016.

[18] M. Jin, J. Lee, and N. G. Tsagarakis, "Model-free robust adaptive control of humanoid robots with flexible joints," IEEE Trans. Indus. Electron., vol. 64, no. 2, pp. 1706-1715, 2017.

[19] S. Roy, I. N. Kar, J. Lee, N. G. Tsagarakis, and D. G. Caldwell, "Adaptive-robust control of a class of EL systems with parametric variations using artificially delayed input and position feedback," IEEE Trans. Control Syst. Technol., vol. 27, no. 2, pp. 603-615, 2019.

[20] J. Lee, H. Dallali, M. Jin, D. Caldwell, and N. Tsagarakis, "Robust and adaptive whole-body controller for humanoids with multiple tasks under uncertain disturbances," in IEEE International Conference on Robotics and Automation, 2016, pp. 5683-5689.

[21] J. Lee, H. Dallali, M. Jin, D. G. Caldwell, and N. G. Tsagarakis, "Robust and adaptive dynamic controller for fully-actuated robots in operational space under uncertainties," Autonomous Robots, vol. 43, no. 4, pp. 1023-1040, 2019

[22] M. Pi, Y. Kang, C. Xu, G. Li, and Z. Li, "Adaptive time-delay balance control of biped robots," IEEE Trans. Indus. Electron., vol. 67, no. 4 pp. 2936-2944, 2019.

[23] S. Roy, J. Lee, and S. Baldi, "A new continuous-time stability perspective of time-delay control: Introducing a state-dependent upper bound structure," IEEE Control Syst. Lett., vol. 3, no. 2, pp. 475-480, 2019.

[24] S. Roy, S. Baldi, and L. M. Fridman, "On adaptive sliding mode control without a priori bounded uncertainty," Automatica, vol. 111, p. 108650,2020

[25] S. Roy, S. B. Roy, J. Lee, and S. Baldi, "Overcoming the underestimation and overestimation problems in adaptive sliding mode control," IEEE/ASME Trans. Mechatron., vol. 24, no. 5, pp. 2031-2039, 2019.

[26] S. Roy, J. Lee, and S. Baldi, "A new adaptive-robust design for time delay control under state-dependent stability condition," IEEE Trans. Control Syst. Technol., vol. 29, no. 1, pp. 420-427, 2020.

[27] $\mathrm{Z}$. Yu et al., "Humanoid walking pattern generation based on the ground reaction force features of human walking," in IEEE International Conference on Information and Automation, 2012, pp. 753-758. 